Supporting Information

\title{
Synergistic electrocatalytic hydrogen evolution in Ni/NiS nanoparticles \\ wrapped in multi-heteroatom-doped reduced graphene oxide nanosheets
}

Mohamed Barakat Zakaria Hegazy, ${ }^{1,2,3 *}$, Mohamed Reda Berber, ${ }^{4}$ Yusuke Yamauchi, ${ }^{5,6}$ Amir Pakdel, ${ }^{7}$ Rui Cao, ${ }^{8}$ and Ulf-Peter Apfel ${ }^{1,2 *}$

1. Inorganic Chemistry I, Faculty for Chemistry and Biochemistry, Ruhr University Bochum, 44801 Bochum, Germany.

2. Fraunhofer Institute for Environmental, Safety and Energy Technology UMSICHT, 46047 Oberhausen, Germany.

3. Department of Chemistry, Faculty of Science, Tanta University, 31527 Tanta, Egypt.

4. Chemistry Department, College of Science, Jouf University, Sakaka 2014, Saudi Arabia.

5. JST-ERATO Yamauchi Materials Space-Tectonics Project and International Research Center for Materials Nanoarchitechtonics (WPI-MANA), National Institute for Materials Science (NIMS), 1-1 Namiki, Tsukuba, Ibaraki 305-0044, Japan.

6. School of Chemical Engineering and Australian Institute for Bioengineering and Nanotechnology (AIBN), The University of Queensland, Brisbane, Queensland 4072, Australia.

7. Department of Mechanical, Manufacturing \& Biomedical Engineering, Trinity College Dublin, Dublin D02PN40, Ireland.

8. Key Laboratory of Applied Surface and Colloid Chemistry, Ministry of Education, School of Chemistry and Chemical Engineering, Shaanxi Normal University, Xi'an 710119, China.

Emails: MBZ (mohamed.hegazy3@science.tanta.edu.eg) and UPA (ulf.apfel@rub.de) 
Figure S1
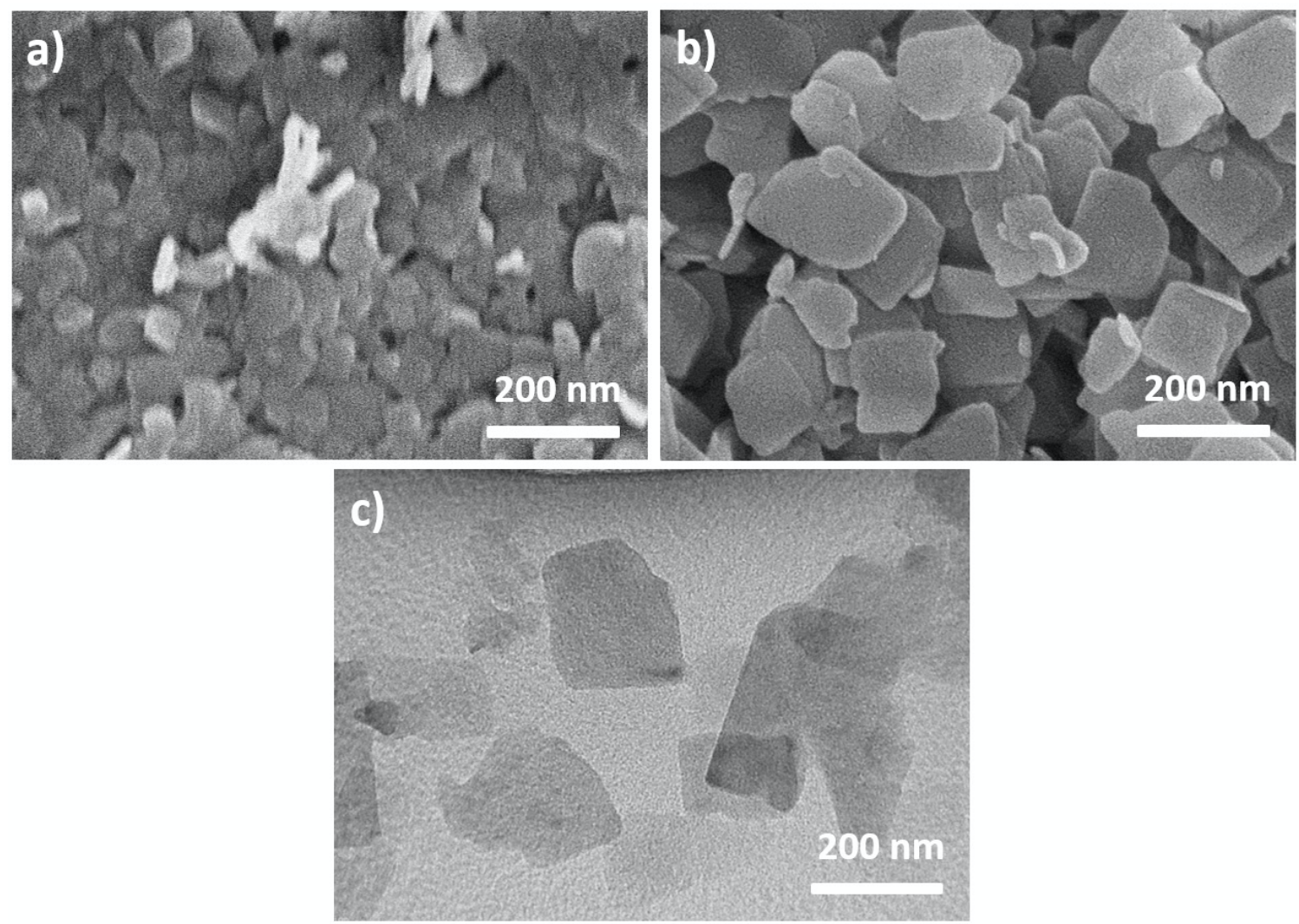

Figure S1 a) SEM image of NiCNNi random particles prepared without using TSCD and b,c) SEM and TEM images of NiCNNi flakes prepared using TSCD as a controlling agent.

Figure S2
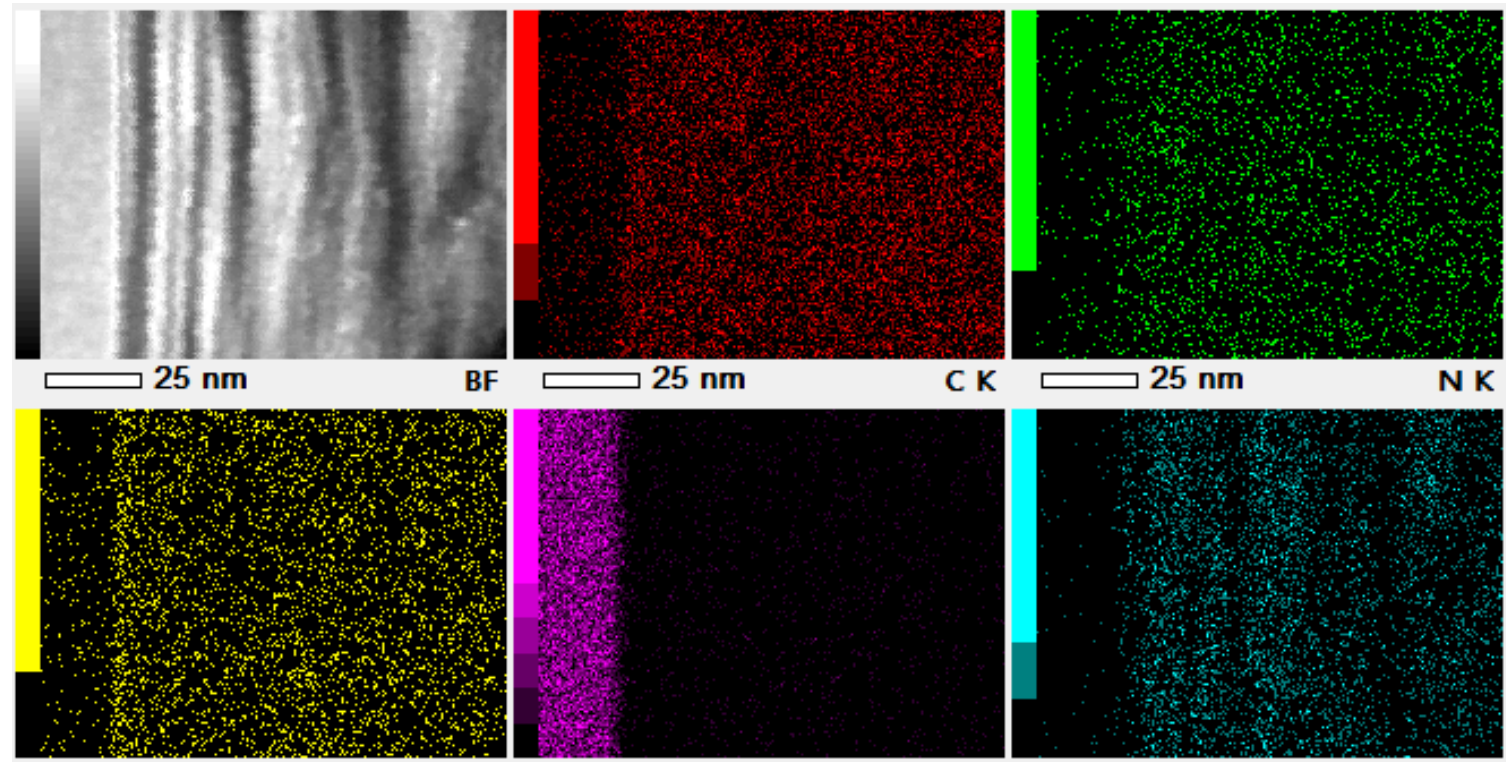

$\square 25 \mathrm{~nm}$

C K

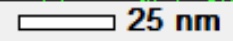

N K

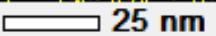

O K
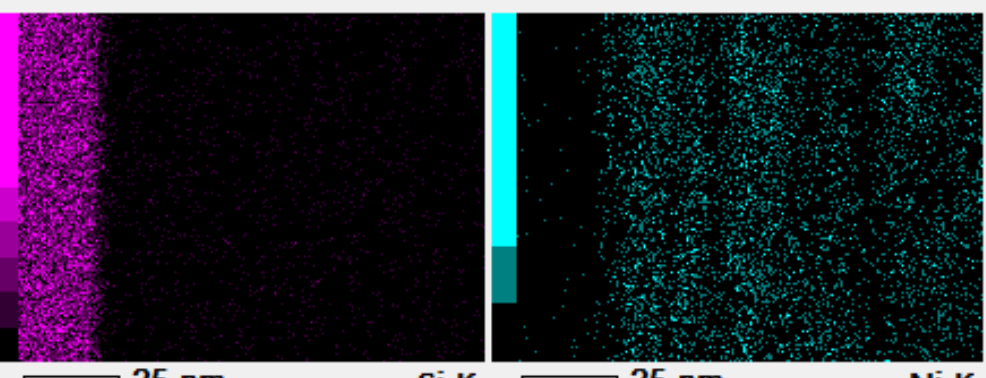

Figure S2 Cross-sectional HAADF-STEM and the corresponding atom mappings of NiCNNi-GO hybrid. 
Figure S3

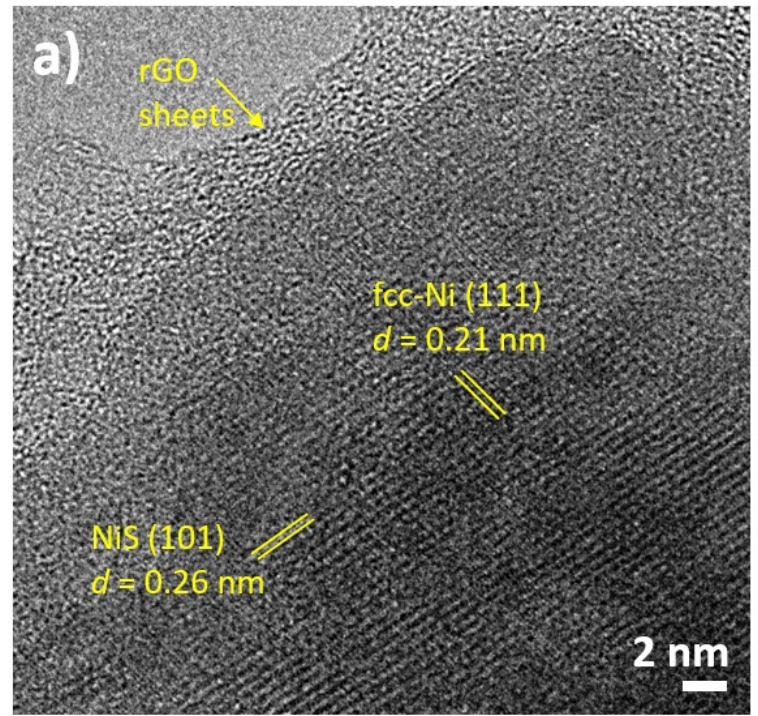

\section{b)}

Figure S3 a) HRTEM and b) SAED images of Ni/NiS/P,N,S-rGO heterostructure.

Figure S4
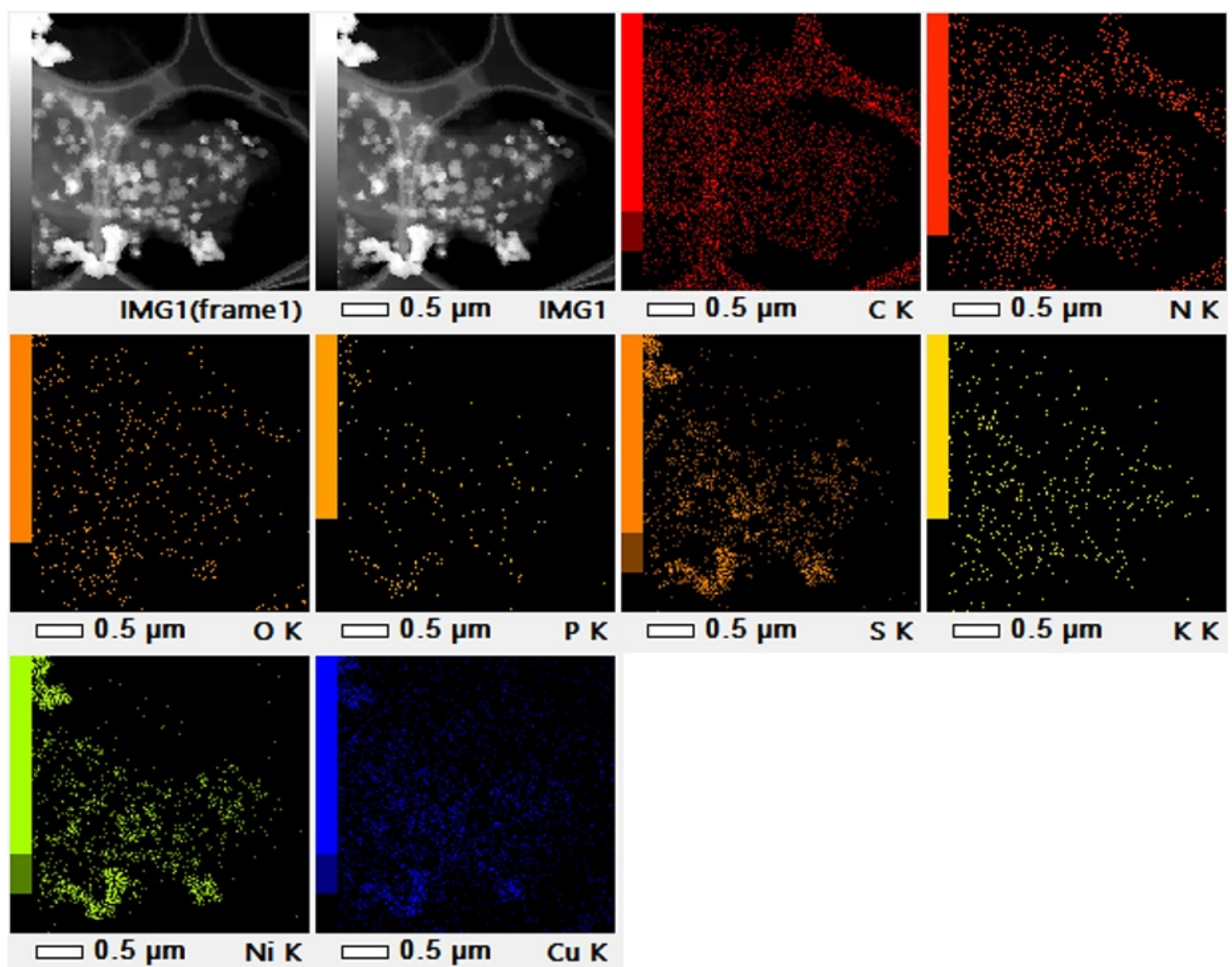

K K

Figure S4 Top-view HAADF-STEM and the corresponding atom mappings of Ni/NiS/P,N,S-rGO heterostructure. 
Figure S5

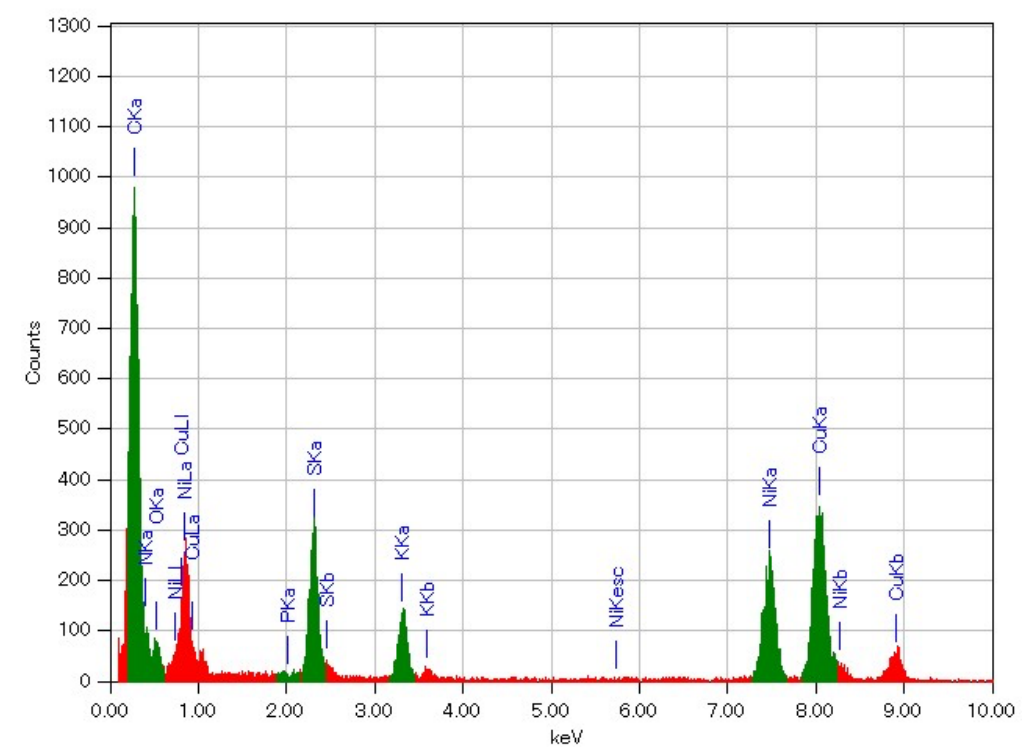

Figure S5 EDS spectra attached to TEM of Ni/NiS/P,N,S-rGO heterostructure.

Figure S6
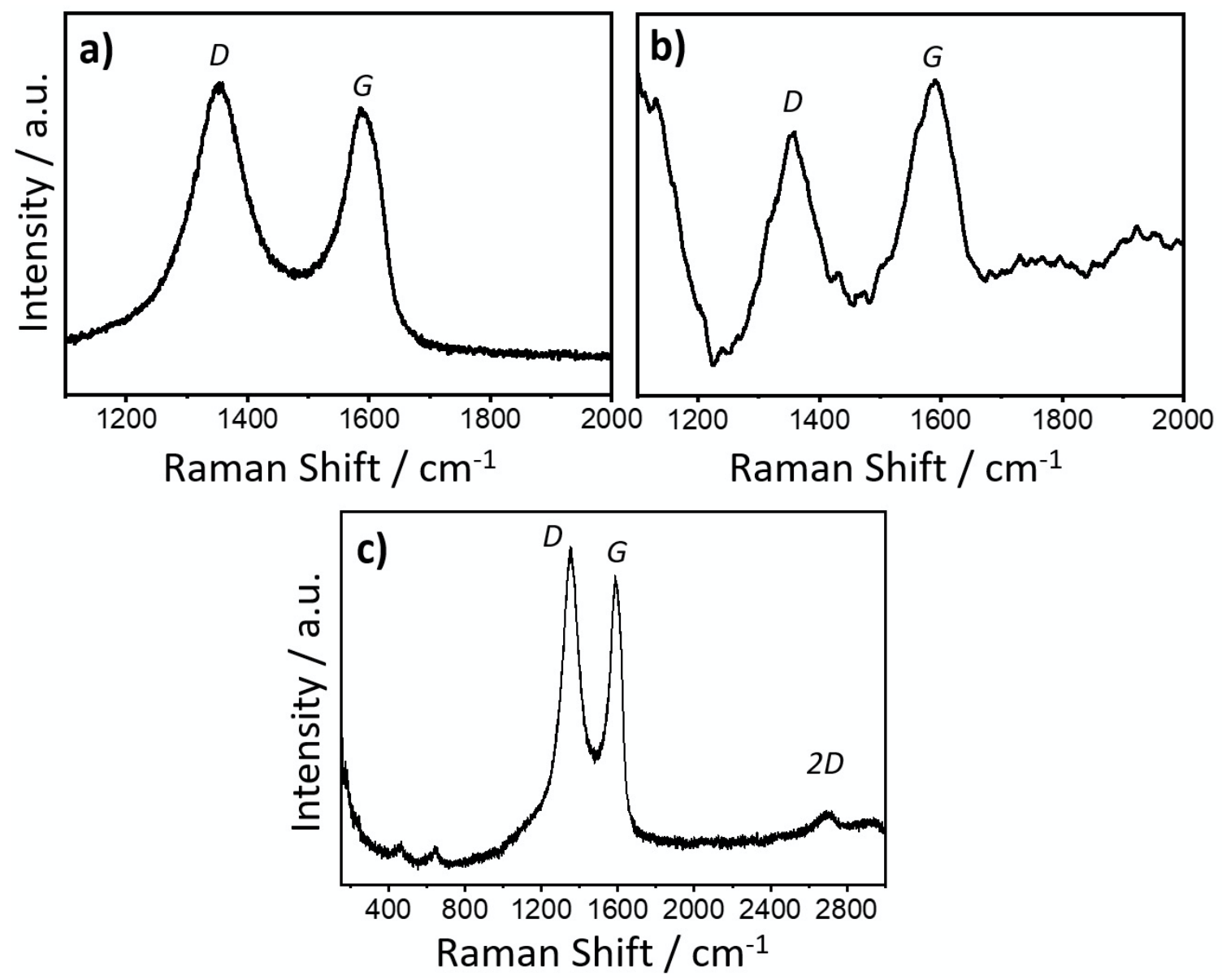

Figure S6 Raman spectra of a) the as-prepared GO nanosheets and b) annealed rGO sheets at $450{ }^{\circ} \mathrm{C}$ in nitrogen. c) Wide survey Raman spectrum of GO sheets to determine $2 D$ band. 
Figure S7

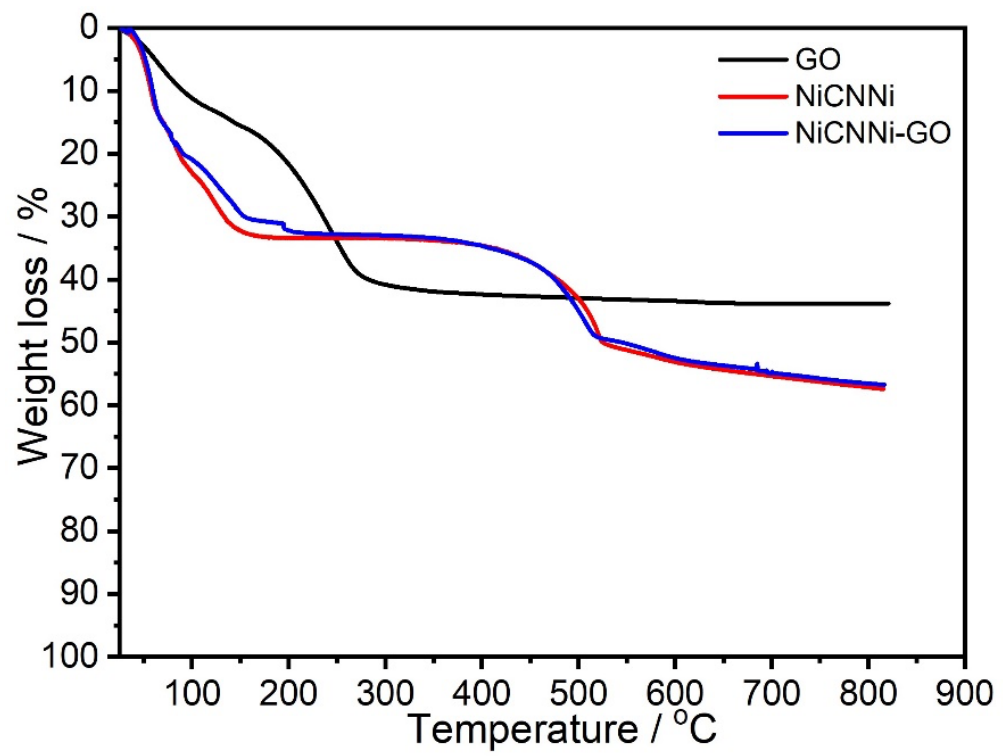

Figure S7 TGA curves of GO sheets, NiCNNi flakes, and NiCNNi-GO hybrid samples at a heating rate of $5{ }^{\circ} \mathrm{C}$ $\mathrm{min}^{-1}$ from room temperature to $800{ }^{\circ} \mathrm{C}$ under a $\mathrm{N}_{2}$ gas flow rate of $120 \mathrm{~mL} \mathrm{~min}^{-1}$.

Figure S8

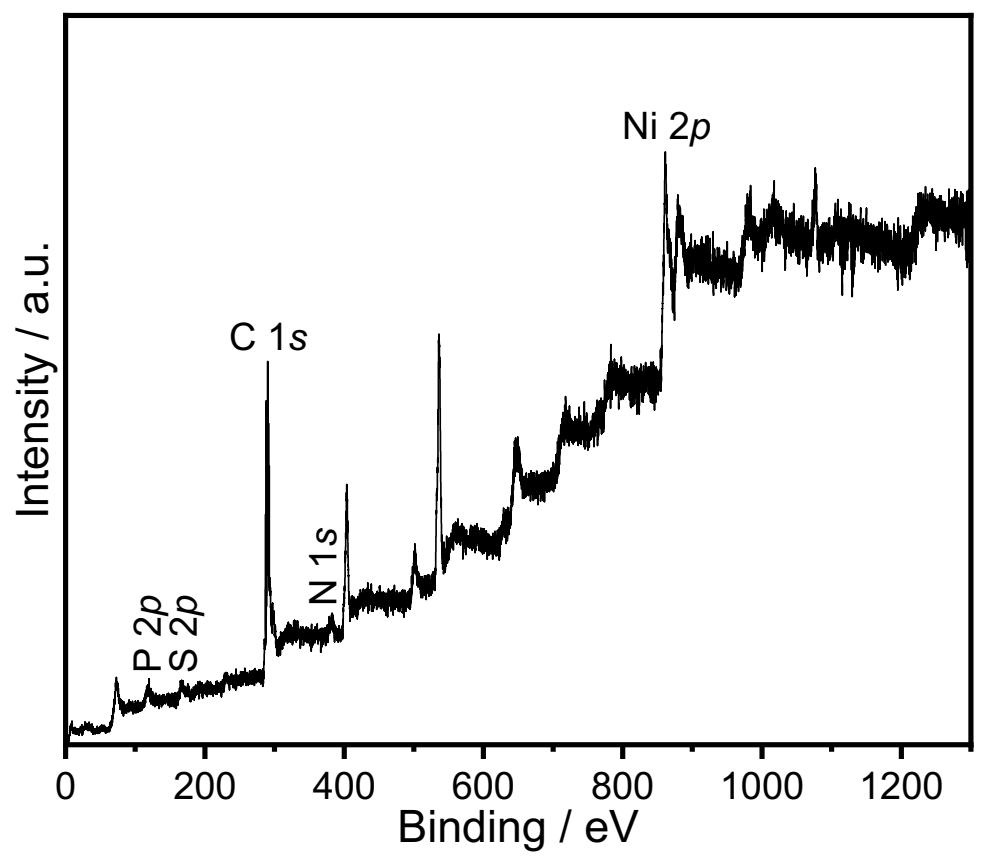

Figure S8 Overall XPS survey spectrum recorded on Ni/NiS/P,N,S-rGO heterostructure. 
Figure S9
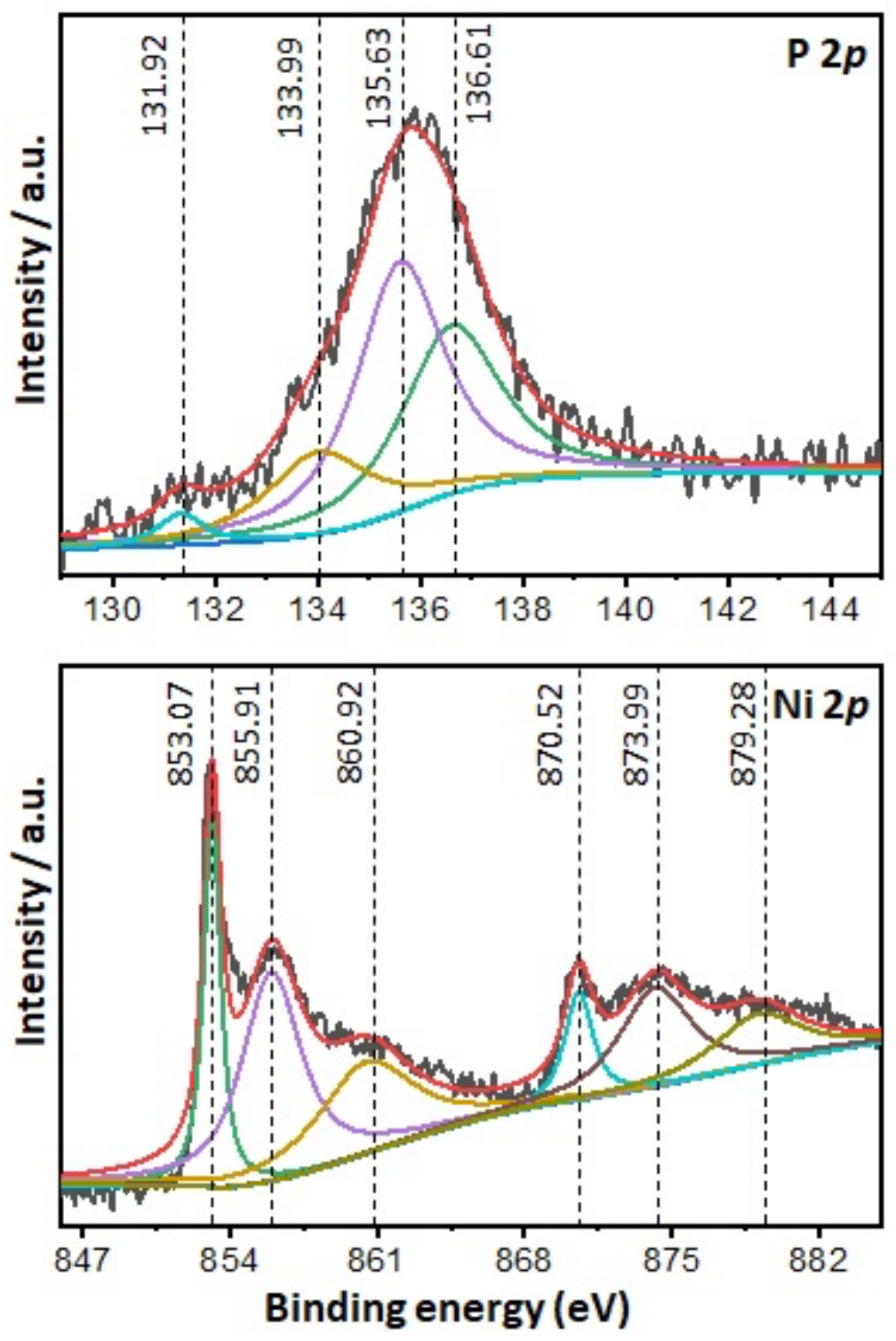

Figure S9 High resolution hard X-ray photoelectron spectra (HAXPES) of P $2 p$ and Ni $2 p$ orbitals recorded on $\mathrm{Ni} / \mathrm{NiS} / \mathrm{P}, \mathrm{N}, \mathrm{S}-\mathrm{rGO}$ heterostructure. 
Figure S10
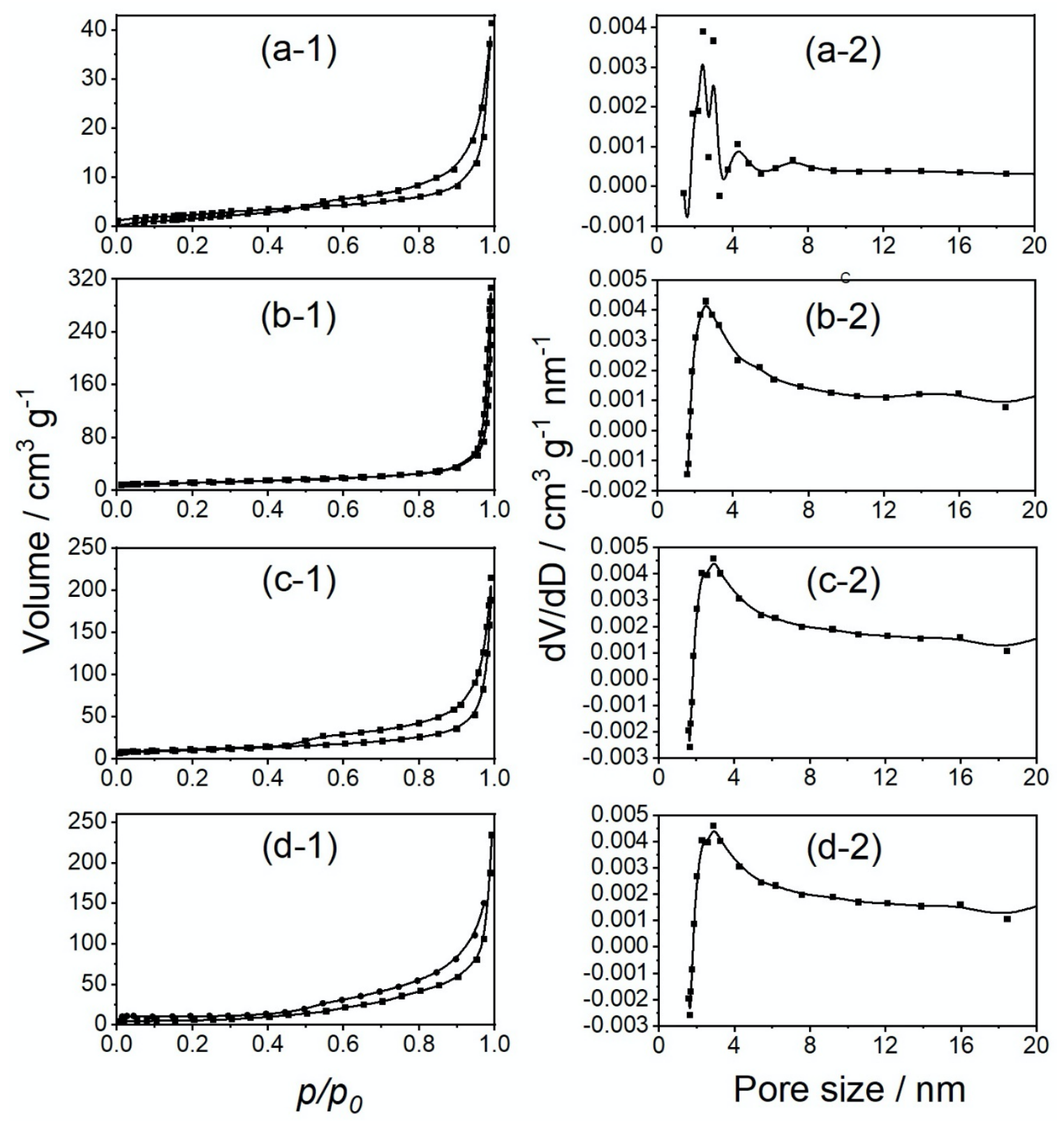

Figure S10 Nitrogen gas adsorption-desorption isotherms and pore size distribution of a) $\mathrm{rGO}$, b) $\mathrm{NiS}$, c) $\mathrm{Ni}_{3} \mathrm{C} / \mathrm{P}-$ rGO, and Ni/NiS/P,N,S-rGO samples. 
Figure S11

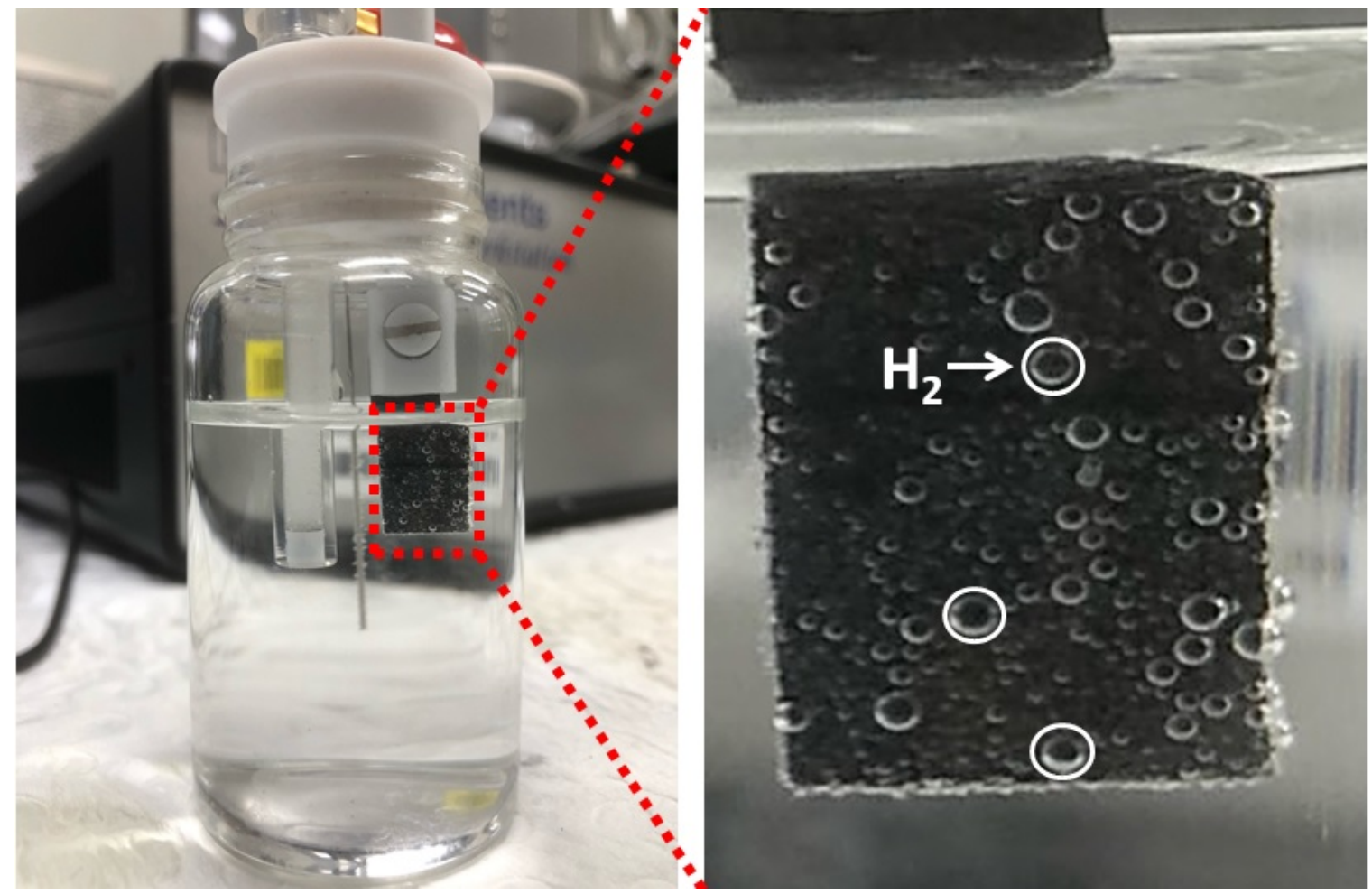

Figure 11 Photographs of three-electrode cell set up and the working electrode while $\mathrm{H}_{2}$ evolutes.

Figure S12

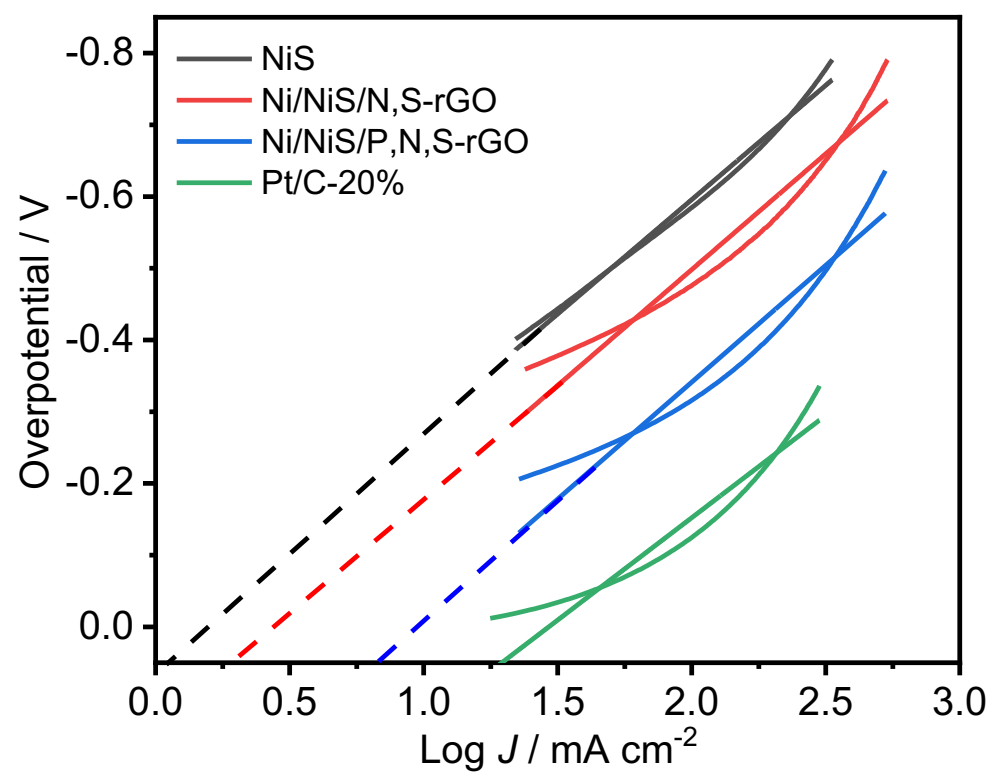

Figure S12 Determination of exchange current density $\left(j_{0}\right)$ by Tafel extrapolation method. 
Figure S13
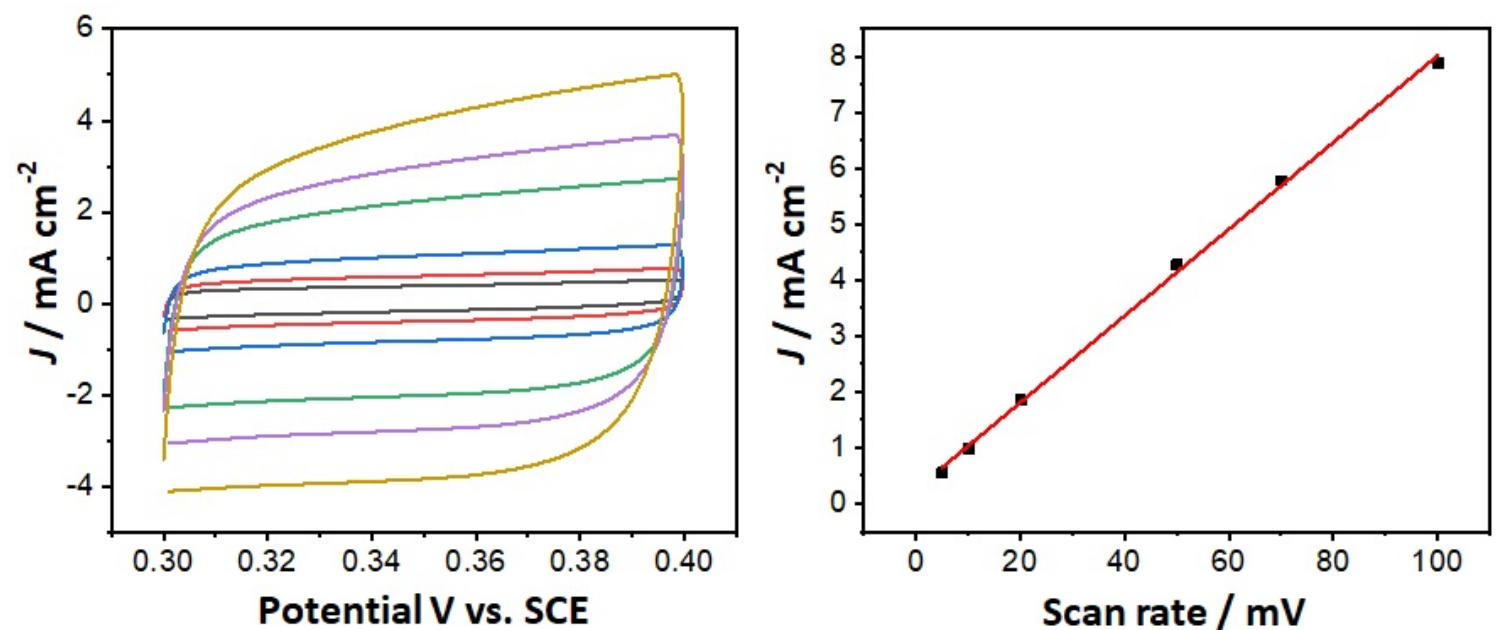

Figure S13 Static cyclic voltammetry curves (CVs) at 5.0, 10, 20, 50, 70 and $100 \mathrm{~V} \mathrm{~s}^{-1}$ and dependence of double layer charging current $v s$. scan rate plots of $\mathrm{Ni} / \mathrm{NiS} / \mathrm{P}, \mathrm{N}, \mathrm{S}-\mathrm{rGO}$ composite electrode.

Figure 14
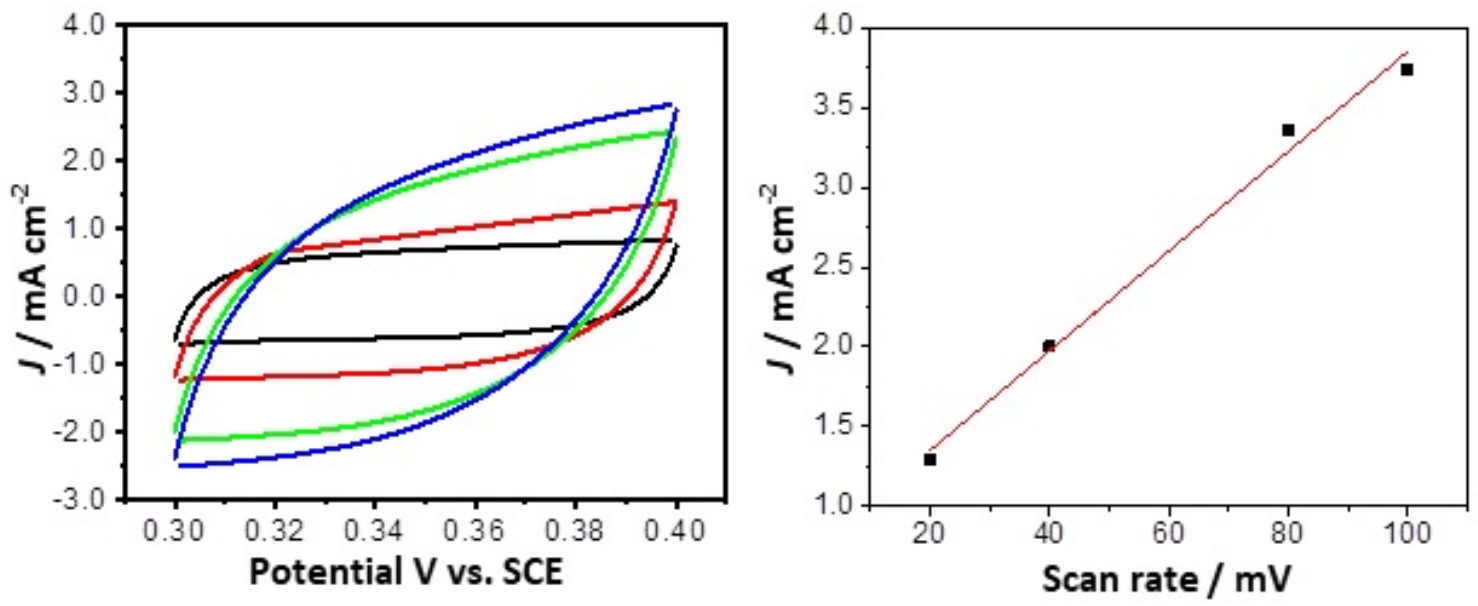

Figure S14 Static cyclic voltammetry curves (CVs) at 20, 40, 80 and $100 \mathrm{~V} \mathrm{~s}^{-1}$ and dependence of double layer charging current $v s$. scan rate plots of $\mathrm{Ni} / \mathrm{NiS} / \mathrm{N}, \mathrm{S}-\mathrm{rGO}$ composite electrode. 
Figure S15
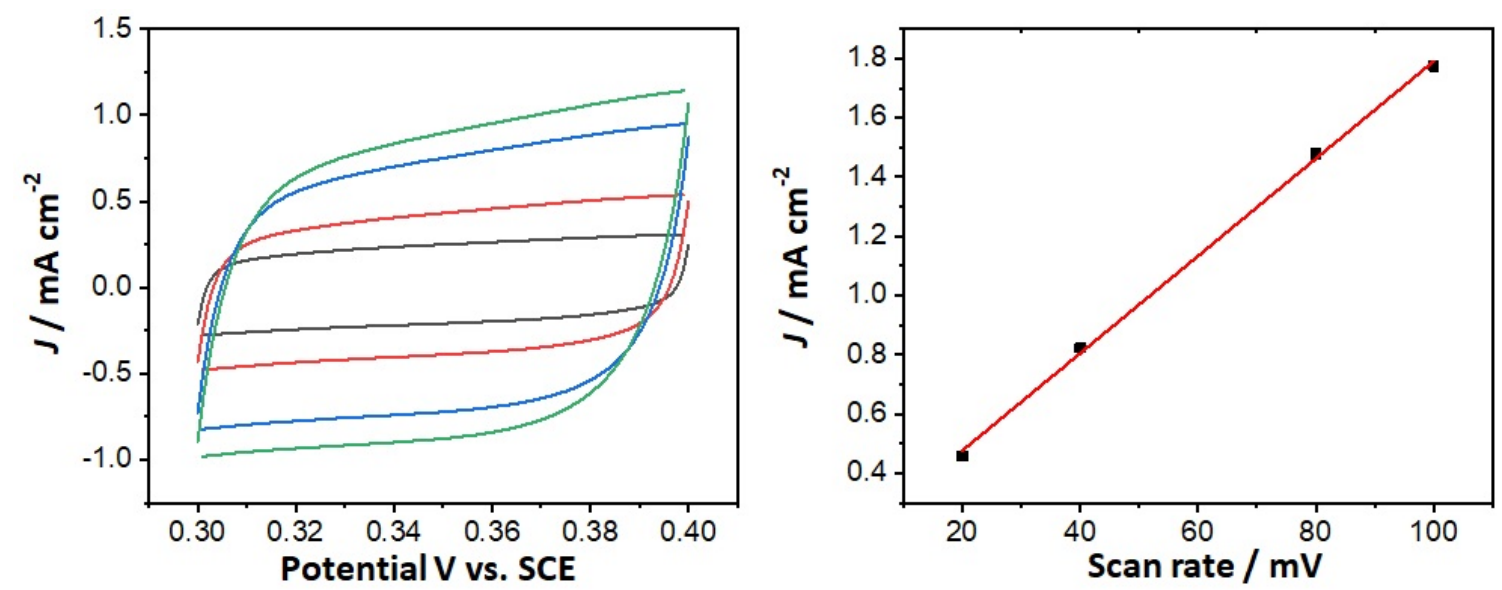

Figure S15 Static cyclic voltammetry curves (CVs) at 20, 40, 80 and $100 \mathrm{~V} \mathrm{~s}^{-1}$ and dependence of double layer charging current vs. scan rate plots of NiS electrode.

Figure S16
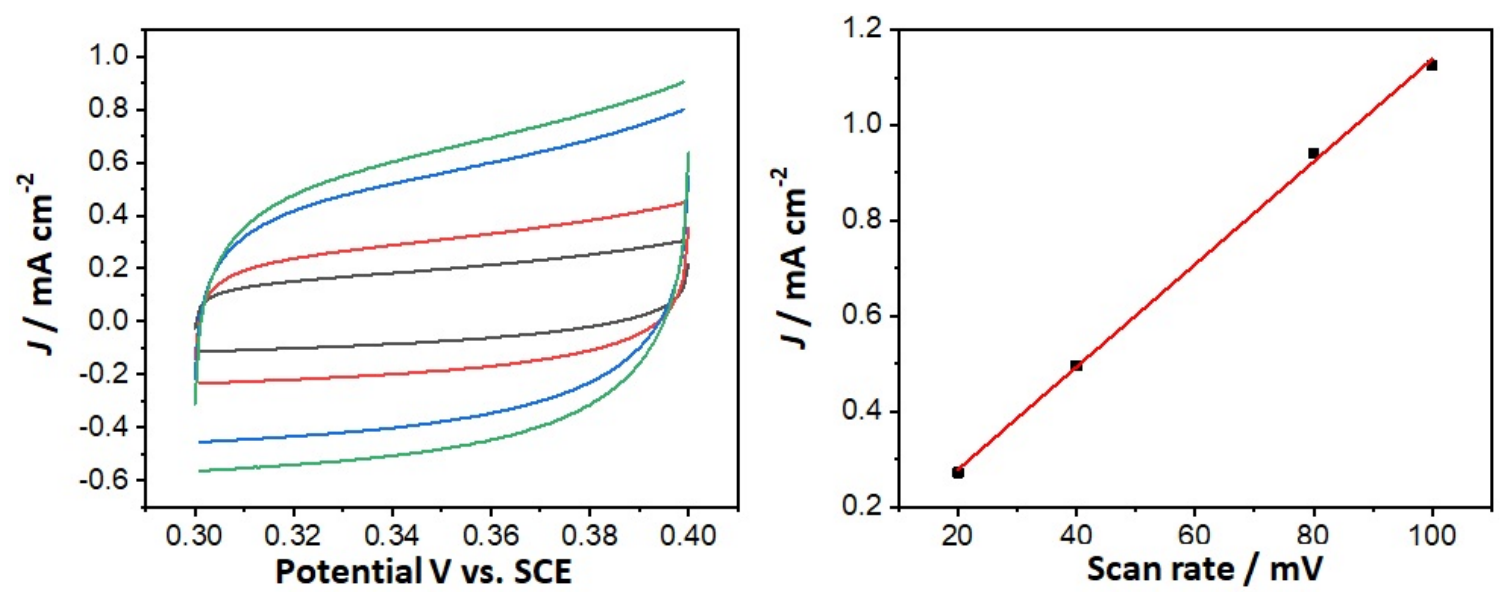

Figure S16 Static cyclic voltammetry curves (CVs) at 20, 40, 80 and $100 \mathrm{~V} \mathrm{~s}^{-1}$ and dependence of double layer charging current vs. scan rate plots of rGO electrode. 
Figure S17

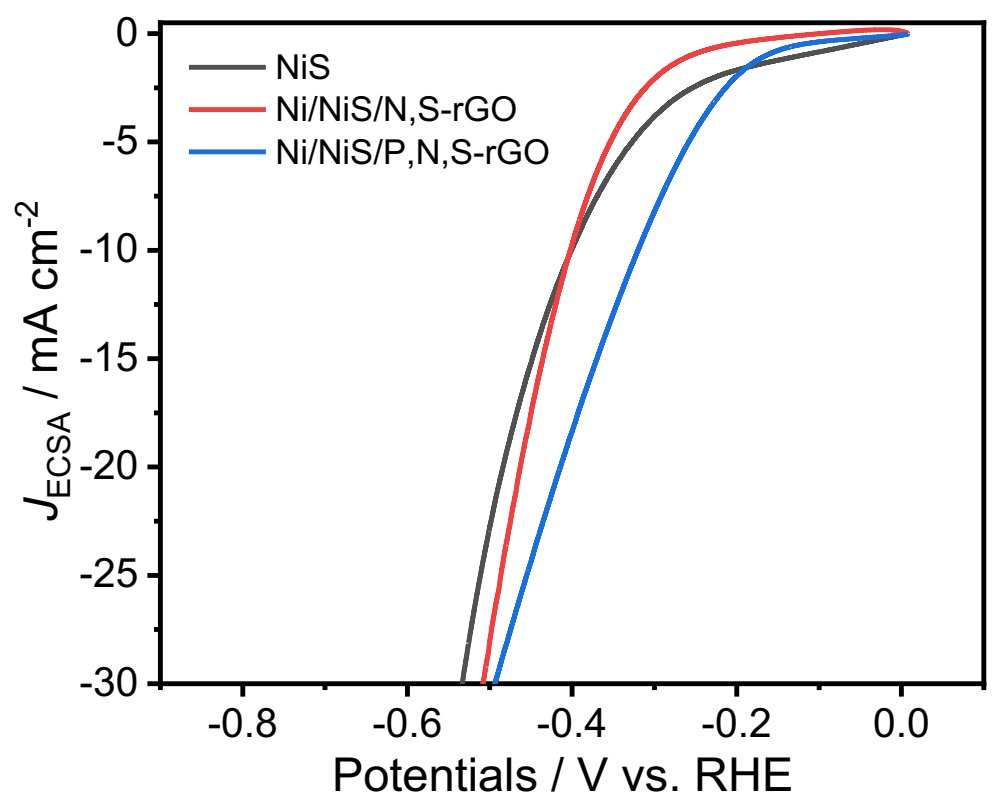

Figure S17 LSV profiles for NiS, Ni/NiS/N,S-rGO and Ni/NiS/P,N,S-rGO electrodes that are normalized to the ECSA.

Figure 18

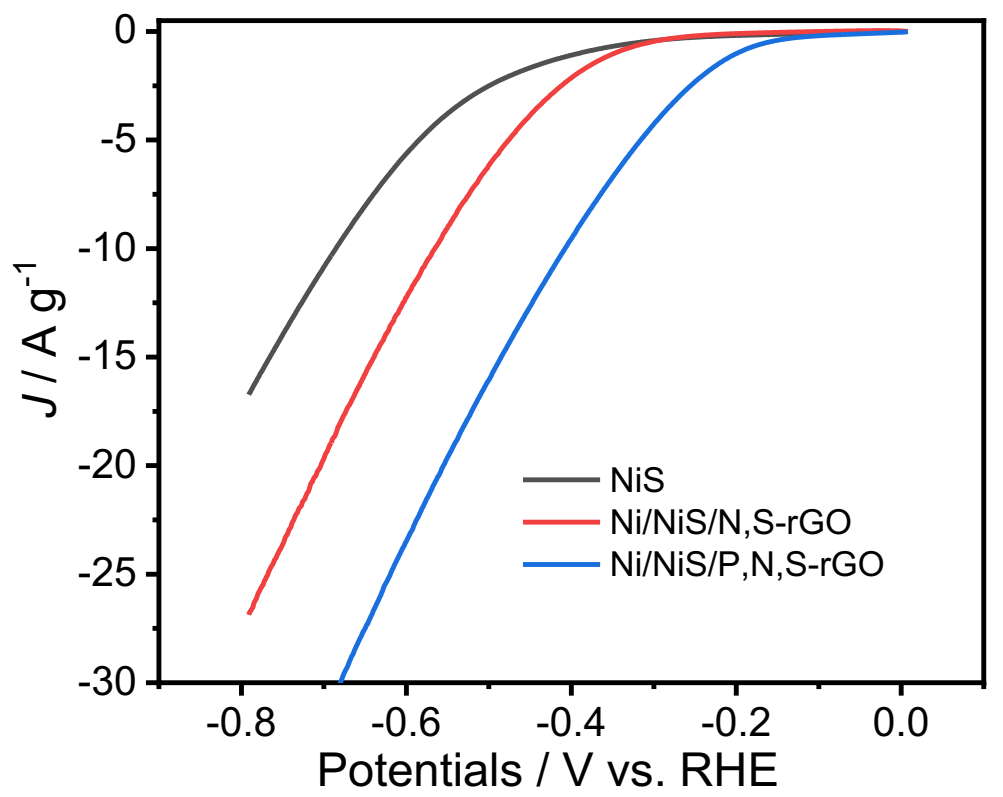

Figure S18 LSV profiles for NiS, Ni/NiS/N,S-rGO and Ni/NiS/P,N,S-rGO electrodes that are normalized to the mass loading. 
Figure S19

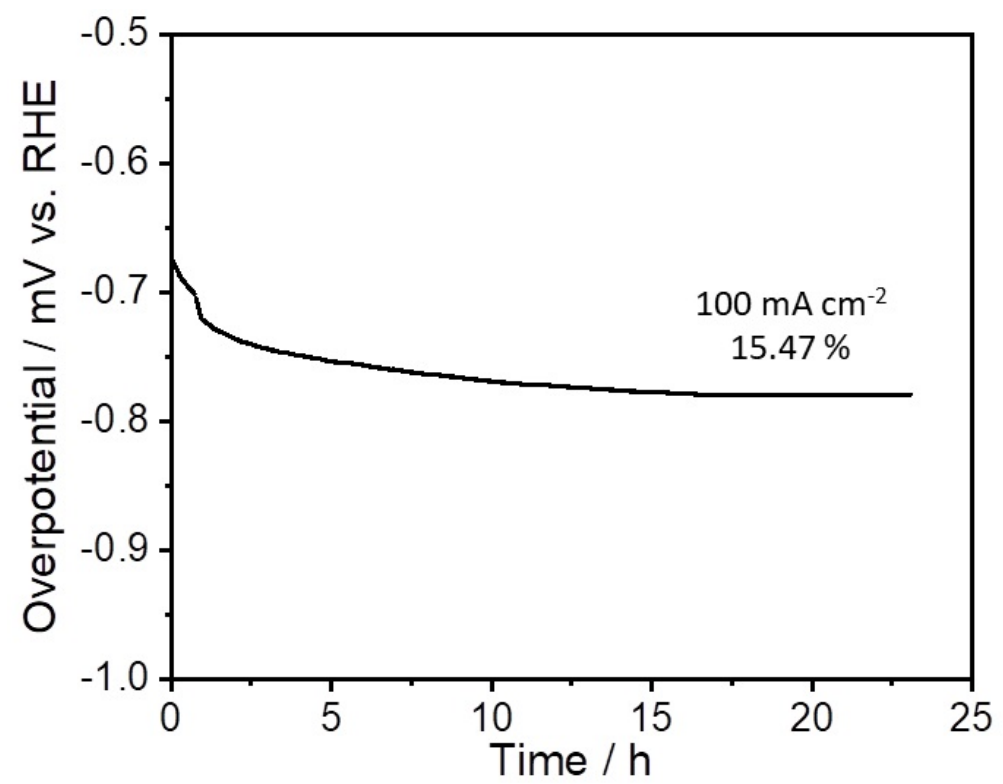

Figure S19 Long term stability testing of the Ni/NiS/P,N,S-rGO composite electrode through chronopotentiometry measurements at a constant current density of $100 \mathrm{~mA} \mathrm{~cm}^{-2}$.

Figure S20

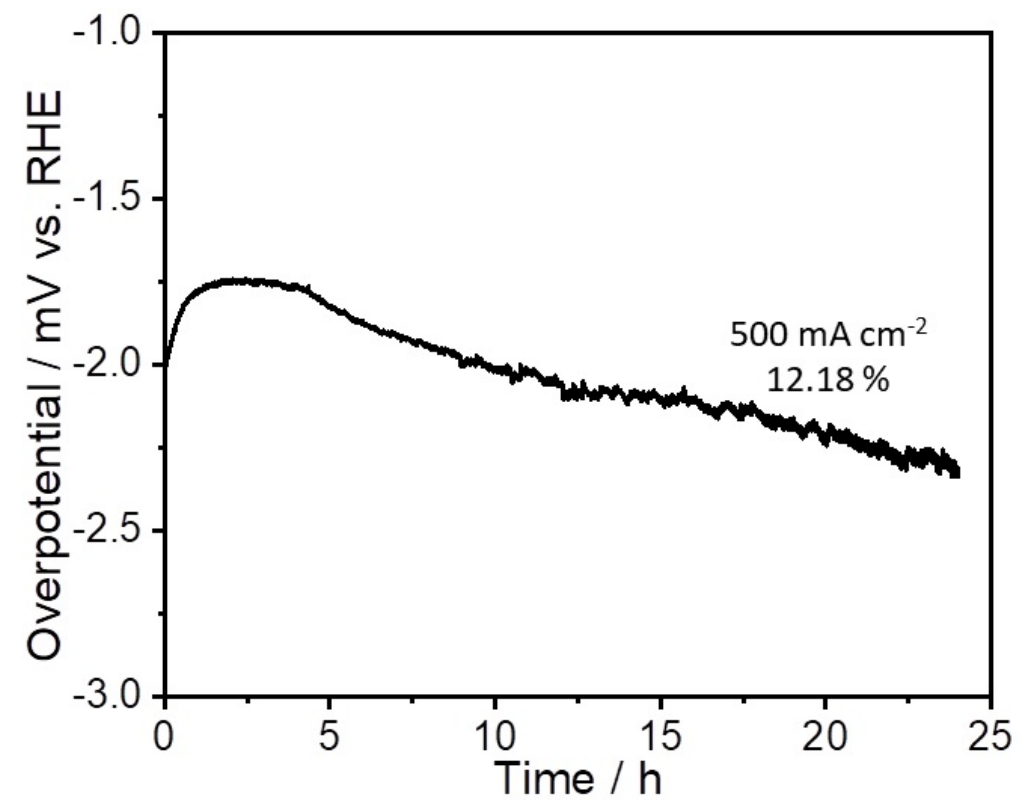

Figure S20 Long term stability testing of the Ni/NiS/P,N,S-rGO composite electrode through chronopotentiometry measurements at a constant current density of $100 \mathrm{~mA} \mathrm{~cm}^{-2}$. 
Figure S21
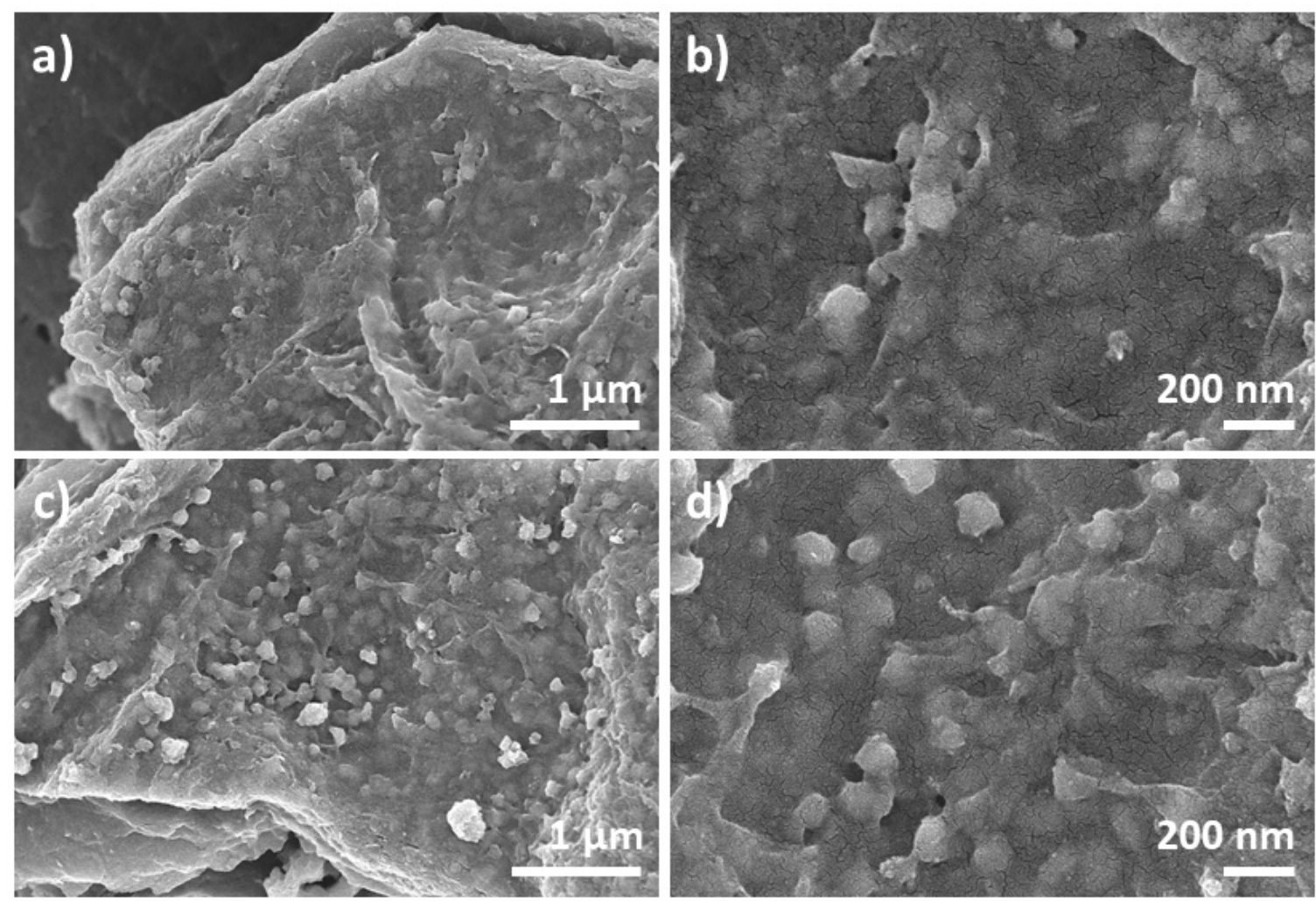

Figure S21 Low and high magnification SEM images of a,b) as-prepared and c,d) used Ni/NiS/P,N,S-rGO heterostructured electrode.

Figure S22

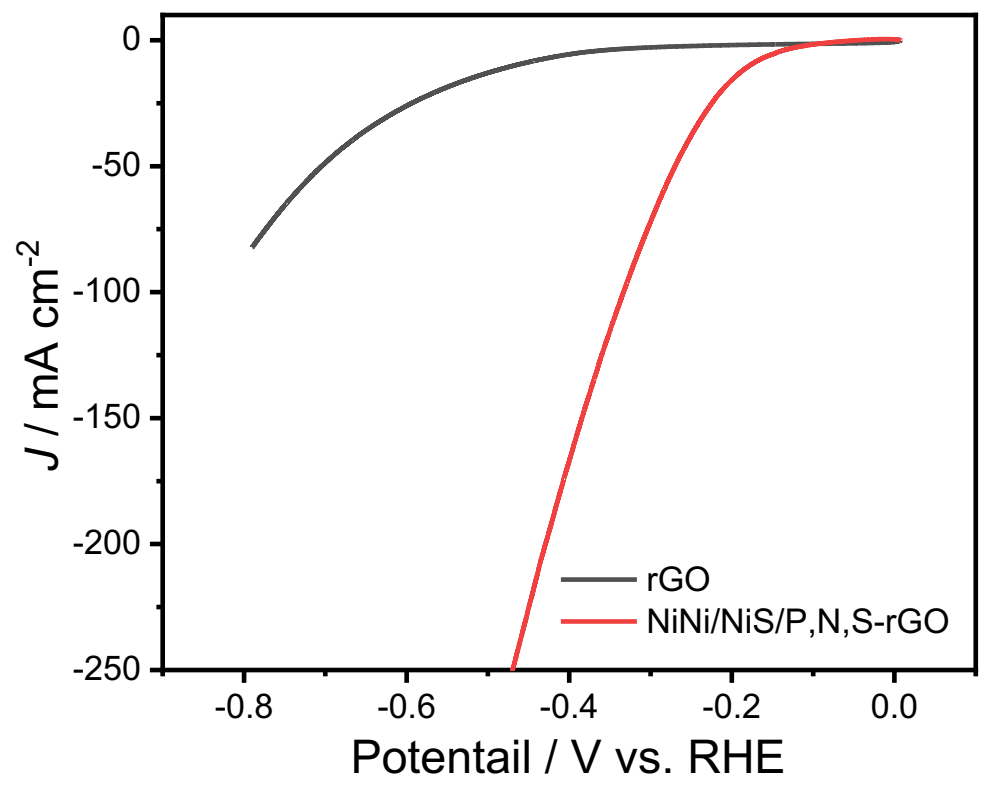

Figure S22 LSV curves at a scan rate of $50 \mathrm{mV} \mathrm{S}^{-1}$ in $1.0 \mathrm{~m} \mathrm{KOH}$ solution of rGO and Ni/NiS/P,N,S-rGO electrodes. 
Figure S23

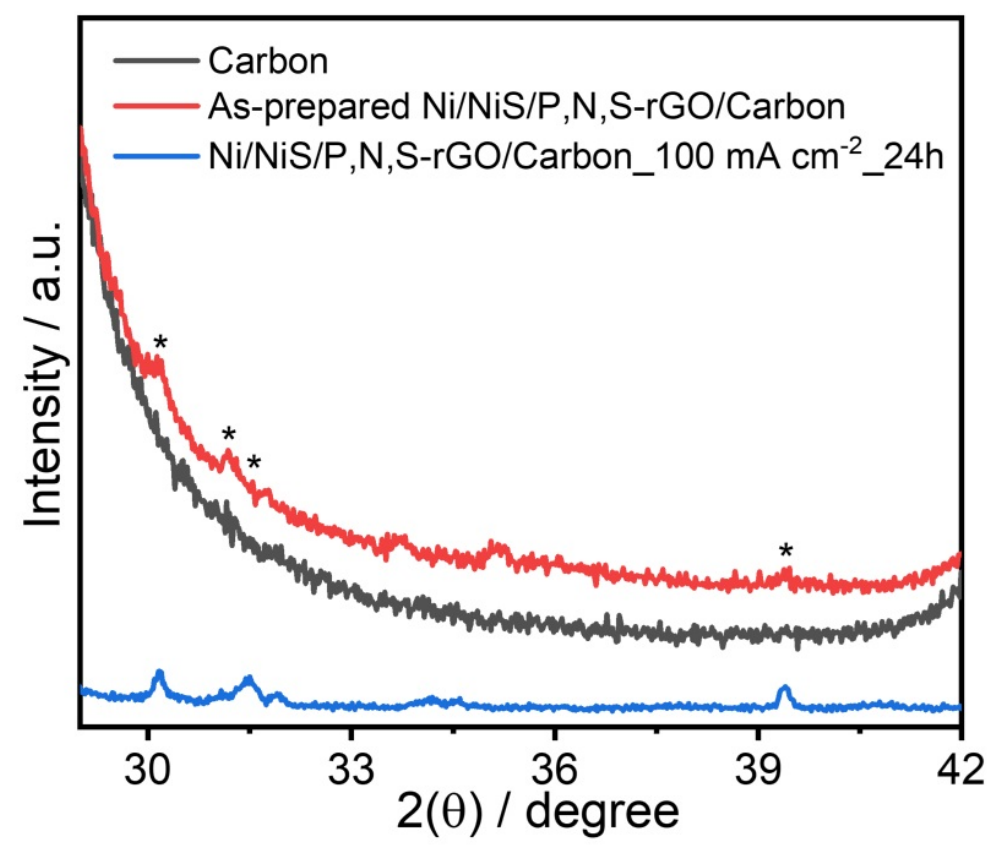

Figure S23 Wide-angle XRD patterns of carbon and as-prepared Ni/NiS/P,N,S-rGO/carbon electrodes and $\mathrm{Ni} / \mathrm{NiS} / \mathrm{P}, \mathrm{N}, \mathrm{S}-\mathrm{rGO}$ electrode after chronopotentiometry at $100 \mathrm{~mA} \mathrm{~cm}{ }^{-2}$ for $24 \mathrm{~h}$. 

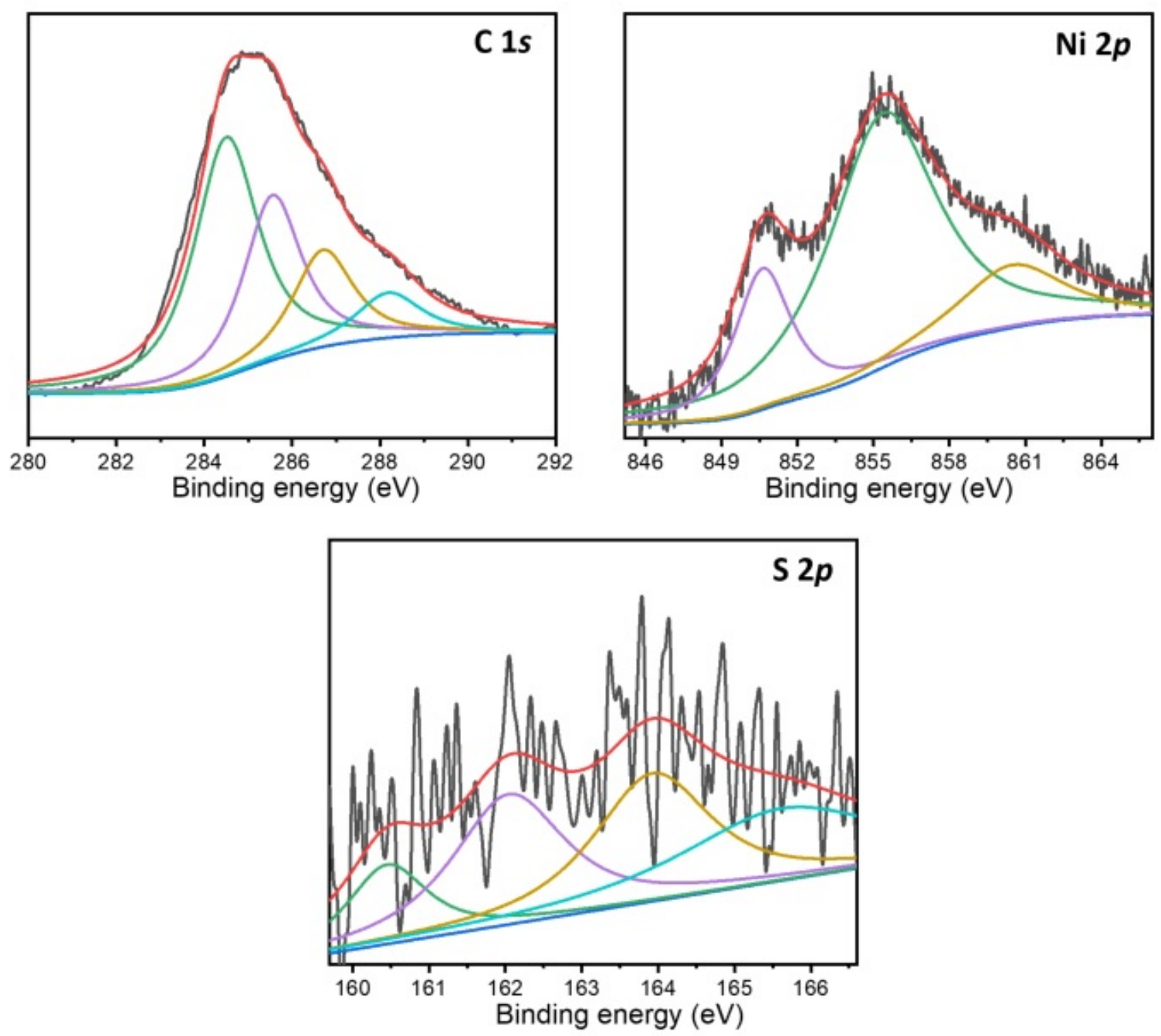

Figure S24 High resolution X-ray photoelectron spectra (XPS) of Ni $2 p, \mathrm{C} 1 s$, and S $2 p$ orbitals recorded on $\mathrm{Ni} / \mathrm{NiS} / \mathrm{P}, \mathrm{N}, \mathrm{S}-\mathrm{rGO} /$ carbon electrode after long term chronopotentiometry test at $100 \mathrm{~mA} \mathrm{~cm}^{-2}$.

Table S1. Comparison of the electrocatalytic performance of Ni/NiS/P,N,S-rGO toward HER with other electrocatalysts previously demonstrated.

\begin{tabular}{|c|c|c|c|c|c|}
\hline Catalysts & $\begin{array}{l}\text { Current density } \\
\quad\left(j, \mathrm{~mA} \mathrm{~cm}^{-2}\right)\end{array}$ & $\begin{array}{c}y_{10} \\
(\mathrm{mV})\end{array}$ & $\begin{array}{l}\text { Tafel slope } \\
\left(\mathrm{mV} \cdot \mathrm{dec}^{-1}\right)\end{array}$ & Electrolytes & References \\
\hline Ni/NiS/P,N,S-rGO & 10 & 155 & 135 & $1.0 \mathrm{M} \mathrm{KOH}$ & This work \\
\hline Hollow-Fe-CoMoS & 10 & 138 & 98.0 & $1.0 \mathrm{M} \mathrm{KOH}$ & S1 \\
\hline Ni@NC-800 & 10 & 205 & 160 & $1.0 \mathrm{M} \mathrm{KOH}$ & $\mathrm{S} 2$ \\
\hline $\mathrm{CoS}_{2}$ & 10 & 233 & 150.2 & $1.0 \mathrm{M} \mathrm{KOH}$ & S3 \\
\hline $\operatorname{Co}\left(\mathbf{S}_{0.82} \mathrm{Se}_{0.18}\right)_{2}$ & 10 & 180 & 132.5 & $1.0 \mathrm{M} \mathrm{KOH}$ & S3 \\
\hline $\mathrm{Co}\left(\mathrm{S}_{0.71} \mathrm{Se}_{0.29}\right)_{2}$ & 10 & 122 & 85.7 & $1.0 \mathrm{M} \mathrm{KOH}$ & $\mathrm{S} 3$ \\
\hline $\mathrm{Co}\left(\mathrm{S}_{0.47} \mathrm{Se}_{0.53}\right)_{2}$ & 10 & 128 & 103.2 & $1.0 \mathrm{M} \mathrm{KOH}$ & $\mathrm{S} 3$ \\
\hline $\mathrm{Co}\left(\mathrm{S}_{0.22} \mathrm{Se}_{0.78}\right)_{2}$ & 10 & 175 & 117.9 & $1.0 \mathrm{M} \mathrm{KOH}$ & S3 \\
\hline $\mathrm{CoSe}_{2}$ & 10 & 175 & 133.5 & $1.0 \mathrm{M} \mathrm{KOH}$ & S3 \\
\hline
\end{tabular}




\begin{tabular}{|c|c|c|c|c|c|}
\hline $\mathrm{CogS}_{8} @ \mathrm{MoS}_{2} / \mathrm{CNFs}$ & 10 & 190 & 110 & $1.0 \mathrm{M} \mathrm{KOH}$ & $\mathrm{S} 4$ \\
\hline $\mathrm{MoS}_{2}-\mathrm{Ni}_{3} \mathrm{~S}_{2} \mathbf{H N R s} / \mathbf{N F}$ & 10 & 98.0 & 61.0 & $1.0 \mathrm{M} \mathrm{KOH}$ & S5 \\
\hline $\mathrm{Co}_{3} \mathrm{~S}_{4} @ \mathrm{MoS}_{2}$ & 10 & 136 & 74.0 & $1.0 \mathrm{M} \mathrm{KOH}$ & S6 \\
\hline $\mathrm{Ni} / \mathrm{Ni}_{3} \mathrm{C} / \mathrm{C}-\mathrm{NCNT}$ & 10 & 184 & 98.7 & $1.0 \mathrm{M} \mathrm{KOH}$ & S7 \\
\hline N-doped NiMoS & 10 & 68.0 & 86.0 & $1.0 \mathrm{M} \mathrm{KOH}$ & S8 \\
\hline $\mathrm{Ni}_{3} \mathrm{~S}_{2}$ & 10 & 335 & 97.0 & $1.0 \mathrm{M} \mathrm{KOH}$ & S9 \\
\hline $\mathrm{NiS}_{2}$ & 10 & 454 & 124 & $1.0 \mathrm{M} \mathrm{KOH}$ & S9 \\
\hline NiS & 10 & 474 & 128 & $1.0 \mathrm{M} \mathrm{KOH}$ & S9 \\
\hline MoNiS@NiS/CC & 10 & 68.0 & 130 & $1.0 \mathrm{M} \mathrm{KOH}$ & S10 \\
\hline Hollow-NiMo3 $\mathrm{S}_{4}$ & 10 & 59.0 & 98.0 & Ar- $0.1 \mathrm{M} \mathrm{KOH}$ & S11 \\
\hline $\mathrm{S}-\mathrm{CoSe}_{2} / \mathrm{GC}$ & 10 & 102 & 52.0 & $0.5 \mathrm{M} \mathrm{H}_{2} \mathrm{SO}_{4}$ & S12 \\
\hline $\mathbf{O}-\mathrm{Ni}_{3} \mathrm{~S}_{2}$ & 10 & 68.4 & 60.6 & $1.0 \mathrm{M} \mathrm{KOH}$ & S13 \\
\hline $\mathrm{C}-\mathrm{Ni}_{3} \mathrm{~S}_{2}$ & 10 & 85.5 & 73.04 & $1.0 \mathrm{M} \mathrm{KOH}$ & S13 \\
\hline $\mathrm{Cog}_{8} \mathrm{~S}_{8}-60$ & 10 & 178 & 82.0 & $0.5 \mathrm{M} \mathrm{H}_{2} \mathrm{SO}_{4}$ & S14 \\
\hline Nanoporous-CogS8 & 10 & 264 & 118 & $1.0 \mathrm{M}$ PBS & S15 \\
\hline Nanoporous-Cog $\mathbf{S}_{4} \mathbf{P}_{4}$ & 10 & 87.0 & 51.0 & $1.0 \mathrm{M}$ PBS & $\mathrm{S} 15$ \\
\hline meso-Fe-MoS $/ \mathrm{CoMo}_{2} \mathrm{~S}_{4}$ & 10 & 122 & 90.0 & $1.0 \mathrm{M} \mathrm{KOH}$ & $\mathrm{S} 16$ \\
\hline
\end{tabular}

\section{References}

S1. Guo, Y.; Zhou, X.; Tang, J.; Tanaka, S.; Kaneti, Y. V.; Na, J.; Jiang, B.; Yamauchi, Y.; Bando, Y.; Sugahara, Y. Multiscale Structural Optimization: Highly Efficient Hollow Iron-Doped Metal Sulfide Heterostructures as Bifunctional Electrocatalysts for Water Splitting. Nano Energy 2020, 75 , 104913.

S2. $\quad$ Xu, Y.; Tu, W.; Zhang, B.; Yin, S.; Huang, Y.; Kraft, M.; Xu, R. Nickel Nanoparticles Encapsulated in Few-Layer Nitrogen-Doped Graphene Derived from Metal-Organic Frameworks as Efficient Bifunctional Electrocatalysts for Overall Water Splitting. Adv. Mater. 2017, 29, 1605957.

S3. $\quad$ Fang, L.; Li, W.; Guan, Y.; Feng, Y.; Zhang, H.; Wang, S.; Wang, Y. Tuning Unique Peapod-Like $\mathrm{Co}\left(\mathrm{S}_{\mathrm{x}} \mathrm{Se}_{1-\mathrm{x}}\right)_{2}$ Nanoparticles for Efficient Overall Water Splitting. Adv. Funct. Mater. 2017, 27, 1701008.

S4. Zhu, H.; Zhang, J.; Yanzhang, R.; Du, M.; Wang, Q.; Gao, G.; Wu, J.; Wu, G.; Zhang, M.; Liu, B.; Yao, J.; Zhang, X. When Cubic Cobalt Sulfide Meets Layered Molybdenum Disulfide: A Core-Shell System Toward Synergetic Electrocatalytic Water Splitting. Adv. Mater. 2015, 27, 4752-4759.

S5. Yang, Y.; Zhang, K.; Lin, H.; Li, X.; Chan, H. C.; Yang, L.; Gao, Q. MoS $2-\mathrm{Ni}_{3} \mathrm{~S}_{2}$ Heteronanorods as Efficient and Stable Bifunctional Electrocatalysts for Overall Water Splitting. ACS Catal. 2017, 27, $2357-2366$.

S6. Guo, Y.; Tang, J.; Wang, Z.; Kang, Y.-M.; Bando, Y.; Yamauchi, Y. Elaborately Assembled Core-Shell Structured Metal Sulfides as a Bifunctional Catalyst for Highly Efficient Electrochemical Overall Water Splitting. Nano Energy 2018, 47, 494-502.

S7. Dong, T.; Zhang, X.; Cao, Y.; Chen, H.-S.; Yang, P. Ni/Ni ${ }_{3}$ C Core-Shell Nanoparticles Encapsulated in N-doped Bamboo-like Carbon Nanotubes towards Efficient Overall Water Splitting. Inorg. Chem. Front. 2019, 6, 1073-1080. 
S8. Huang, C.; Yu, L.; Zhang, W.; Xiao, Q.; Zhou, J.; Zhang, Y.; An, P.; Zhang, J.; Yu, Y. N-doped Ni-Mo Based Sulfides for High-efficiency and Stable Hydrogen Evolution Reaction, Appl. Catal. B Environ. 2020, 276, 119137.

S9. Jiang, N.; Tang, Q.; Sheng, M.; You, B.; Jiang, D.; Sun, Y. Nickel Sulfides for Electrocatalytic Hydrogen

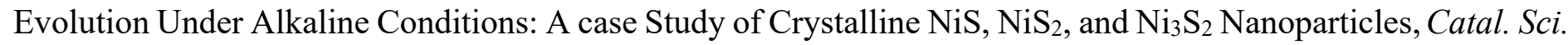
Technol. 2016, 6, 1077-1084.

S10. Xie, Y.; Liu, Y.; Yang, Z. Interfaces Engineering of MoNi-based Sulfides Electrocatalysts for Hydrogen Evolution Reaction in Both Acid and Alkaline Media, Int. J. Hydrogen Energ. 2020, 45, 6500-6507.

S11. Jiang, J.; Gao, M.; Sheng, W.; Yan, Y. Hollow Chevrel-Phase $\mathrm{NiMo}_{3} \mathrm{~S}_{4}$ for Hydrogen Evolution in Alkaline Electrolytes, Angew. Chem. Int. Ed. 2016, 55, 15240-15245.

S12. Xue, N.; Lin, Z.; Li, P.; Diao, P.; Zhang, Q. Sulfur-Doped CoSe 2 Porous Nanosheets as Efficient Electrocatalysts for the Hydrogen Evolution Reaction, ACS Appl. Mater. Interfaces 2020, 12, 28288-28297.

S13. Zheng, X.; Zhang, L.; Huang, J.; Peng, L.; Deng, M.; Li, L.; Li, J.; Chen, H.; Wei, Z. Boosting Hydrogen Evolution Reaction of Nickel Sulfides by Introducing Nonmetallic Dopants, J. Phys. Chem. C 2020, 124, 2422324231.

S14. Yang, Y.; Yuan, M.; Li, H.; Sun, G.; Ma, S. Controllable Synthesis of Ultrathin Co9S 8 Nanosheets as A Highly Efficient Electrocatalyst for Overall Water Splitting, Electrochimica Acta 2018, 281, 198-207.

S15. Tan, Y.; Luo, M.; Liu, P.; Cheng, C.; Han, J.; Watanabe, K.; Chen, M. Three-Dimensional Nanoporous Co9S $4 \mathrm{P}_{4}$ Pentlandite as a Bifunctional Electrocatalyst for Overall Neutral Water Splitting, ACS Appl. Mater. Interfaces 2019, 11, 3880-3888.

S16. Guo, Y.; Tang, J.; Henzie, J.; Jiang, B.; Xia, W.; Chen, T.; Bando, Y.; Kang, Y.-M.; Hossain, M. S. A.; Sugahara, Y.; Yamauchi, Y. Mesoporous Iron-doped $\mathrm{MoS}_{2} / \mathrm{CoMo}_{2} \mathrm{~S}_{4}$ Heterostructures through Organic-Metal Cooperative Interactions on Spherical Micelles for Electrochemical Water Splitting. ACS Nano 2020, 14, 41414152. 